\title{
Niche-Specialist And Age-Related Microbial Ecosystem: Correlation Analysis With Immune Cells In Oral Homeostasis
}

\section{Dongjia Lin}

Sun Yat-Sen University Guanghua School of Stomatology

\section{Lisa Yang}

Sun Yat-Sen University Guanghua School of Stomatology

Qiannan Hu

Sun Yat-Sen University Guanghua School of Stomatology

\section{Dikan Wang}

Sun Yat-Sen University Guanghua School of Stomatology

\section{Wenxiao Dai}

Sun Yat-Sen University Guanghua School of Stomatology

\section{Yiwei Xiao}

Sun Yat-Sen University Guanghua School of Stomatology

Huanzi Lu

Sun Yat-Sen University Guanghua School of Stomatology

\section{Juan Fang}

Sun Yat-Sen University Guanghua School of Stomatology

\section{Zhi Wang ( $\nabla$ wangzh75@mail.sysu.edu.cn )}

Sun Yat-Sen University https://orcid.org/0000-0002-5276-1413

\section{Research}

Keywords: Oral microbial ecosystem, Niche-specialist microbiome, Age-related microbiota, Mucosal microbiology, Oral homeostasis

Posted Date: September 17th, 2021

DOI: https://doi.org/10.21203/rs.3.rs-829179/v1

License: (c) (1) This work is licensed under a Creative Commons Attribution 4.0 International License. Read Full License 


\section{Abstract}

Background: The oral microbial ecosystem, with distinct characteristics in structure and evolution, plays an important role in the mucosal homeostasis. The characterization of baseline microbial and functional diversity in the oral microbiome has been discussed, yet the taxon-taxon relationships, and the role of the microbes themselves to the host mucosa are still lack of discussing. In addition, the current literature seems insufficient to draw a definitive conclusion about a possible impact of ageing on the oral microbiota. Therefore, the study focuses on the spatial and temporal characteristics of the oral microbial ecosystem, and its correlation with immune cell in oral homeostasis.

Results: The V3V4 region of 16S rRNA gene of 30 samples from different sites (gingiva, palate, buccal, tongue) and life stages (adult, old) was analyzed. Flow cytometry was used to investigate the residing immune cells. The niche-specialist and age-related communities, characterized by alterations in microbiota structure, taxon-taxon relationship, microbial functions, and immune cell inhabiting, have been addressed. Interestingly, network analysis shows more antagonistic interactions in gingiva, palatal site, and old mice saliva, where harbor a richer diversity. While in buccal mucosa and tongue, it appears more synergic interactions to maintain the ecosystem balance. Functional analysis reveals that the significant factor that determines the niche for a microbe is its local habitat in the content of one specific age, which includes its immediate neighbors. Correlation analysis suggests strong associations between distinct oral bacteria and Th cells exist in different life stages.

Conclusions: Our findings propose that the oral microbial ecosystem, with niche-specialist and agerelated characteristics, has unique evolution and co-evolution with the host to maintain oral homeostasis, which provides critical insights on mucosal microbiology.

\section{Introduction}

The oral microbial ecosystem, with distinct characteristics in structure and evolution, plays an important role in the mucosal homeostasis[1, 2]. The oral mucosa, where consists of various sites[3], e.g., the gingiva (tooth-associated), buccal (lining), palate (masticatory) and tongue (specialized), is a unique community of complex and dynamics crosstalk with commensal microbiome. This biosystem of microorganisms has a remarkable capacity to adapt to its host with co-evolution[4,5].

50 years ago, Socransky and Manganiello firstly introduced that the oral microbial communities were distinguishable in sites and ages[6]. However, it was not fully confirmed due to the technological limitation. In 2019, Welch et al. proposed the site-specialist hypothesis for oral microbiota[7] and the original concept of micron-scale structure, which defines the microbial habitats and niches in combination with short- and long-range interactions[8]. To date, the characterization of the microbiota in different fields has been recognized by high-throughput identification of microbes with 16S rRNA genes[9-15]. The importance of spatial organization in microbial ecology has been highlighted in various aspects including the gut microbiota[16], the upper respiratory tract[17], and the mouth ecosystem[18- 
21]. Human Microbiome Project (HMP) in distinctive oral habitats has achieved great progress these years $[9,14]$. Though these findings present a broad view of the distinction in human oral habitats, they are currently focused on explaining the role of oral microorganisms presenting in disease[22, 23] rather than that in homeostasis. In addition, the characterization of baseline microbial and functional diversity in the oral microbiome has been discussed, yet the taxon-taxon relationships, and the role of the microbes themselves to the host mucosa are still lack of discussing.

Ageing is a complex, multifaceted process leading to widespread functional decline that affects every organ and tissue[24, 25]. An ageing niche is associated with a wide variety of features at the molecular, cellular and physiological level[26, 27], which also influences the dialog with inherent microorganisms. The risk of oral diseases increases dramatically through the life course[28], to that the microorganisms is argued to have a contribution[2, 29]. However, it is not clear how ecotypes change with the normal ageing process. Given that data regarding the human oral microbiome with ageing is lacking, Yatsunenko et al. has reported this topic on the microbial composition of the human gut microbiome viewed across age and geography[30]. Although we do not have direct proof that the same events occur in the oral cavity, it would not be surprising[5, 31, 32]. Koren et al. interrogate the dynamics of this relationship in the mouth during early life and find that highly tissue-specific responses facilitate maturation[1]. Yet, the current literature seems insufficient to draw a definitive conclusion about a possible impact of ageing on the oral microbiota[31, 33]. A better understanding of the oral microbiota with ageing is a crucial step for the development of better strategies for prevention and treatment of oral diseases.

To withstand a wide variety of microbial and mechanical challenges, the immune system of the oral mucosa is composed of tissue-resident and specially recruited leukocytes[34, 35]. These leukocytes facilitate the establishment and maintenance of local homeostasis but are also capable to cause oral pathologies when are unrestrained[36, 37]. Conventional T cells can be classified as either major histocompatibility class II (MHC II)-restricted and a $\mathrm{T}$ cell receptor (TCR)-expressing $\mathrm{CD} 4^{+} \mathrm{T}$ cells (helper $T$ cells, or TH cells) [38]. Another unique subset of T cells expresses either $\alpha \beta$ or $\gamma \delta$ TCR and mostly express CD8aa homodimers but not CD8 a $\beta$ heterodimers, i.e., unconventional $C D 8 a a^{+} a \beta$ T cells and $\gamma \delta$ T cells[39]. The first encounter of mucosal barriers with the microbiota initiates host-microbiota feedback loops instructing the tailored development of both the immune system and microbiota at each mucosal site. Once established, balanced immunological interactions enable symbiotic relationships with the microbiota in adult life. This process has been extensively investigated in the mammalian monolayer epithelium-covered intestine and lung mucosae[40-43]; however, the mechanisms engaged by the oral mucosa to establish homeostasis lack of discussion[1]. In addition, ageing of the immune system ('immunosenescence') combined with a low-grade inflammation that develops during ageing ('inflammaging') are thought to impact the outcomes of the oral disease challenge from the microbiota[31, 44]. However, the relative importance, mechanistic interrelationships and hierarchical order of these features have not been clarified.

Here, we have compiled an oral microbial community atlas in different oral habitats and life stages. The work entails a comprehensive characterization of microbiome species, their inner interactions, and the 
correlation with mucosal immune cells in oral homeostasis with an age scale, which aims to uncover cell types and functions associated with promoting homeostasis and disease susceptibility at this important barrier. Indeed, we demonstrate that the oral microbial ecosystems are niche-specialist and age-related, not only including the community structure but also the taxon-taxon relationships and the functional role. Furthermore, correlation analysis reveals that the communication between the oral microbiota and tissueresident immune cells (particularly Th cells and $ү \delta ~ T$ cells) involves in oral homeostasis and disease susceptibility. Collectively, the findings provide a microbial census in spatial and temporal scales and suggest a unique microbiota-immune axis mediating mucosal homeostasis, which provides critical insights on mucosal microbiology.

\section{Materials And Methods}

\section{Oral sample preparation}

Wild-type (WT) C57BL/6J mice were maintained in the barrier facility at the Sun Yat-sen University animal center, and all experiments were carried out in accordance with institutional guidelines. The age of mouse was strictly selected as the previous publications[45-51], including 3-month-old mouse as the mature adult group and 18-month-old one as the old group. As for the 3-month-old mice group, the gingiva, palate, tongue, and buccal mucosa were separated from the mice oral cavity[3,52]. The saliva from adult and old groups was collected by Isohelix SK-1 Swabs with Dri-Capsules (Boca Scientific, Boca Raton, FL, USA) according to the previous protocol[53]. Samples were snap frozen in liquid nitrogen and then stored at $-80^{\circ} \mathrm{C}$ until use.

\section{Bacterial DNA extraction, PCR amplification, library preparation and sequencing}

Bacterial DNA was extracted and purified using the PowerSoil kit (Mobio, Carlsbad, CA, USA) for the mucosal samples, while the Isohelix DNA isolation kit (Boca Scientific, Boca Raton, FL, USA) for the saliva samples[53]. The V3V4 regions of the 16S rRNA gene were amplified used specific primer (338F and $806 R$ ) with $12 \mathrm{bp}$ barcode. Sequencing libraries were generated using NEBNext ${ }^{\circledR}$ Ultra ${ }^{\mathrm{TM}}$ II DNA Library Prep Kit for Illumina ${ }^{\circledR}$ (New England Biolabs, MA, USA) following manufacturer's recommendations and index codes were added. The library quality was assessed on the Qubit@ 2.0 Fluorometer (Thermo Fisher Scientific, MA, USA). At last, the library was sequenced on an Illumina Nova6000 platform and 250 bp paired-end reads were generated (Guangdong Magigene Biotechnology Co.,Ltd. Guangzhou, China).

\section{Sequence processing and statistical analysis}

Fastp (version 0.14.1, https://github.com/OpenGene/fastp) was used to control the quality of the Raw Data by sliding window (-W 4 -M 20)[54]. If there were only two groups, student's t-test, Wilcox rank sum test, Wilcoxon's signed rank test or Welch 't-test was selected, but if there were more than two groups, Kruskal Wallis rank sum test or one-way ANOVA was used.

\section{Flow cytometry}


Biopsy preparation into single cell suspension of oral mucosal tissues were performed as previously describe[55]. Data was analyzed with FlowJo software (TreeStar).

Details of materials and methods are provided in the Supplementary Text online.

\section{Results}

\section{Niche-specialist microbial communities in major oral habitats}

Distinct core species in each oral habitat. A total of 989 OTUs (971 thousand sequences in 20 samples) were defined, including 908 OTUs belonging to 651 genera, and 81 OTUs of unclassified genera (Fig. $\mathrm{S} 1 \mathrm{~A}$ ). Alpha- diversity analysis revealed that gingiva and palatal samples were richer (median chao1 index $>250$ ), more diverse (median simpson index $<0.4$ ) and evener (median simpsoneven index $>0.3$ ) than those of tongue and buccal samples respectively (Fig. 1A-C and Fig. S1A, C-D). The coordination of the Bray-curtis PCoA plot (beta-diversity measure) showed that bacterial communities from tongue and buccal samples clustered together, while those from palate and gingival samples formed another two clusters (Fig. 1D). Gingiva and palatal samples harbored a higher relative abundance of Lactobacillus, Staphylococcus, and Streptococcus, while tongue and buccal samples harbored the highest relative abundances of Streptococcus (Fig. 1E-F and Fig. S1B). In particular, the Corynebacterium, Rodentibacter and Stenotrophomonas were proportionally enriched in buccal samples, the Sporosarcina and Atopostipes were proportionally enriched in gingiva, while the Neomicrococcus, Staphylococcaceae and Methylobacterium were enriched in palate (Fig. 1G). Among 989 OTUs identified in different oral sites, 25 were overabundant in gingiva, 57 were overabundant in palate, 21 were overabundant in buccal tissue, and 6 of these taxa were overabundant in tongue (Fig. 1H). We also performed compact visualizations of microbial metagenomes using Graphical phylogenetic analysis (GraPhIAn) of each sample (Fig. S1E-H). All these results suggest the hypothesis that the oral microbiota is site-specialist, and each oral habitat has its distinctive core species[7].

Potential interactions and niche-sharing among oral taxa in different sites. We argue that most microorganisms restricted to the habitat result from the co-evolution of microbes in the unique site leading to highly specific taxon-taxon interplays. Therefore, the network analysis was performed at the top 50 OUT to provide details supporting this hypothesis. Among 216 links identified, there showed negative correlations in gingival and palatal samples (Fig. 2A, D) while a predominance of positive correlations in tongue and buccal samples (Fig. 2B-C). Specifically, fewer links and a lower level of centralization were found in palate (Fig. 2D), whereas more links and a higher level of centralization were found in buccal (Fig. 2C) than in other sample groups. Network analysis was also performed at the genus level (Fig. S2). The results confirm the modular organization of the microbiota, and further accentuate initial findings from the analysis at the level of OTUs.

The functional role in the distinct communities. Not surprisingly, through COG and KEGG pathways analysis (Fig. 2E and Fig. S2E), the potential functional pathways were differently enriched in each oral area. The relative abundance of several metabolic pathway-related OTUs as well as energy production 
and conversion-related OTUs increased in palate samples. In gingiva, there were enriched OTUs associated with cell functional activities like cell wall/membrane/envelope biogenesis, cytoskeleton, intracellular trafficking, secretion, and vesicular transport, cell motility and so on. While in tongue and buccal samples, it was the relative abundance of defense mechanisms-related, replication, recombination and repair-related, and cell cycle control, cell division, chromosome partitioning-related OTUs developed. The findings firstly suggest that the functional role that the specific members play in each oral mucosa presents differently and provides a strong microbiological barrier to support mucosal homeostasis.

The distribution of Th cells and $y \delta T$ cells in different oral sites. Flow cytometry analysis showed that the proportion of Th1 cells in tongue (38.18\%, [27.03\%-49.34\%]) and buccal (18.62\%, [12.99\%-24.24\%] samples was higher than those in gingiva $(7.79 \%,[4.92 \%-10.67 \%])$ and palate $(7.19 \%,[3.62 \%-10.76 \%], P<$ 0.01 , Fig. 3A). The proportion of $\gamma \delta T$ cells in buccal (25.82\%, [22.1\%-29.54\%]) and gingiva $(20.42 \%$, [18.31\%-22.53\%]) samples was higher than those in palate $(14.18 \%,[11.69 \%-16.67 \%])$ and tongue $(9.38 \%$, [5.38\%-13.37\%], $P<0.01$, Fig. 3E). Meanwhile, the proportion of Th2, Th17, Treg cells showed no significance in each oral site (Fig. 3B-D).

Correlation analysis between immune cells and the oral microbiomes. Th1 cells revealed a negative correlation with some opportunity infectious bacterial such as Acinetobacter, Massilia, Aeromonas, Micrococcus and Enterobacter, and some strictly anaerobic or facultative anaerobic bacterial such as genus Staphylococcus, Lactobacillus, Sporosarcina, Atopostipes, Faecalibaculum and Bifidobacterium while those positively correlated with Treg cells (Fig. 4A). Th1 cells showed a negative correlation with the diversity of oral microbiota $\left(R^{2}=0.58, P<0.05\right.$, Fig. 4B-C). From the recent findings, there is no significant correlation between Th2, Th17, $\gamma \delta$ T cells and oral communities in different oral habitats (Fig. 4A-B, D-G). These works arise the potential correlation between the site-specialist microbiota and the distribution of mucosal immune cells, till their causal relationship need more further study.

\section{Age-related microbiome communities in the major life stages}

Distinctive core species in each life stage. A total of 638 OTUs (709 thousand sequences in 10 samples) were defined, including 451 OTUs belonging to 265 genera, and 187 OTUs of unclassified genera (Fig. S3A). Our findings revealed that the community richness was higher in old mice samples than that in adult samples $(P<0.05$, Fig. 5A, Fig. S3A-F), while community diversity and evenness showed no significant difference $(P<0.05$, Fig. $5 \mathrm{~B}-\mathrm{C})$. The coordination of the Bray-curtis PCoA plot (beta-diversity measure) performed two clustering groups (Fig. 5D). GraPhIAn was used to visualize the microbial metagenomes of each sample (Fig. 5E-F). From bar graph representing the relative community composition at the genus level (Fig. 5G, Fig. S3G), genus Streptococcus was the most general microbiome in oral cavity. LEfSe analysis as well as the richness heatmap discovered that the Actinocrinis puniceicyclus, Actinospica, Actinospicaceae and Lactobacillus were proportionally enriched in old samples, while the Jeotgalicoccus, Bosea and Sphingomonas were significantly enriched in adult samples (Fig. 5H-I, Fig. S3I). These data suggests that species abundance, rather than the overall species 
diversity, largely contributes to the observed differences in salivary microbiota between the adult and old groups.

Taxa-taxa interactions in different periods. Among 139 links identified, there showed negative correlations in old samples while a half of positive correlations in adult samples (Fig. 6A-B). Specifically, fewer links and a lower level of centralization were found in the old (Fig. 6B), whereas more links and a higher level of centralization were found in the adult (Fig. 6A). In addition, adult samples contained several healthassociated taxa (Bifidobacterium, Bradyrhizobium, Lactobacillus, Turicibacter, and Corynebacterium; Fig. 6A and Fig. S4A), while disease-associated taxa (Pseudomonas, Enterobacteriaceae, Enterobacter, Staphylococcus, Bacillaceae, Geobacillus, and unclassified genera) were found in old samples (Fig. 6B and Fig. S4B). Network analysis was also performed at the genus level (Fig S4A-B). The analysis at the genus level also readily identified the interactions among specific taxa across the sample groups, such Lactobacillus, Caldalkalibacillus_thermarum, and Bifidobacterium.

The functional role of the oral microbiota in the phases. Differences in predicted COG and KEGG functional pathways were observed in the saliva microbiota of adult and old mice (Fig. 6C and Fig. S4C). Remarkably, increase in metabolic pathways were observed in adult mice, while cell period pathways like cell cycle control, cell division, chromosome partitioning, replication, recombination and repair, were increased in old mice. Taken together, these results suggest that changes of the oral microbiota during ageing and the interference with signaling pathways contribute to the disorders in barrier function and disease susceptibility.

The distribution of Th cells and $\gamma \delta T$ cells in different stages. The findings revealed that the proportion of Th1 cells in adult groups $(28.62 \%$, [21.45\%-35.8\%]) was higher than those in old groups $(11.59 \%$, [9.82\%-13.37\%], $P<0.01$, Fig. 7A, E), while the proportion of Th2 (31.3\%, [16.16\%-46.44\%]), Th17 (27.06\%, [15.76\%-38.36\%]) and Treg (29.74\%, [15.71\%-43.77\%]) T cells in old samples was higher than those in adult samples $(P<0.01$, Fig. 7B-D, F-H). Meanwhile, the proportion of $\mathrm{y} \delta \mathrm{T}$ cells showed no significance between two groups (Fig. S5A-B).

Correlation analysis between immune cells and the oral microbiomes. The correlations of the relative abundance of bacteria with immune cell subsets, including Th1, Th2, Th17, Treg and $\gamma \delta T$ cells, were shown in a spearman correlation heatmap (Fig. 8A). At the genus level, the abundance of old-enriched bacterial positively correlated with the proportion of Th2 (Fig. 8D, $\mathrm{R}^{2}=0.11, P<0.05$ ), Th17 (Fig. 8E, $\mathrm{R}^{2}=$ $0.16, P<0.05)$, Treg (Fig. 8F, $\left.\mathrm{R}^{2}=0.16, P<0.05\right)$ and negatively correlated with the proportion of Th1 (Fig. $\left.8 \mathrm{~B}, \mathrm{R}^{2}=0.19, P<0.05\right)$. In contrast, Sphingomonas, Burkholderia-Caballeronia-Paraburkholderia and Staphylococcus which enriched in adult mice samples, negatively correlated with the proportion of Th2 $\left(R^{2}=0.11, P<0.05\right)$, Th17 $\left(R^{2}=0.16, P<0.05\right)$, and Treg $\left(R^{2}=0.16, P<0.05\right)$ and positively correlated with the proportion of Th1 $\left(R^{2}=0.19, P<0.05\right)$.

\section{Discussion}


Herein we present a robust atlas of the oral microbial ecosystem in different oral habitats and two major life stages, which expands the hypothesis of site-specialist oral microbiota to the niche-specialist and the age-related one. We also explore the taxon-taxon relationship, microbial function and correlation between the oral microbiota and the host immune cells to discover the interactions and the role of the microbes to the host. Our work provides inspiring insights into mucosal microbiology in oral homeostasis[56].

\section{Biogeography of the oral microbiome: the niche-specialist hypothesis}

A strength of our work is the meticulous characterization of oral microbiomes with a strict definition of different oral niches[3]. Four oral mucosal habitats, gingiva as the representative of tooth-associated mucosa, buccal as the representative of lining mucosa, palate as the representative of masticatory mucosa, and tongue as the representative of specialized mucosa[3], are selected to study. Our results support the hypothesis that the oral microbiota distinctly enriches in different oral sites[57], as the previous studies[7-9, 14]. In addition, the oral microbial communities are expected to display a range of communications inside or outside themselves. We next characterized the organization of the complicated interactions by inferring the microbial ecological networks with a random matrix theory (RMT)-based approach[58]. Taxon-taxon dialogs indicate synergic or antagonistic interactions between oral bacteria, as well as their preference for ecological niches[59]. Interestingly, at the top 50 OUT level, it shows more antagonistic interactions in gingival and palatal samples which harbor a rich diversity in communities. On the contrary, buccal and tongue samples occur more synergic interactions to maintain the ecosystem balance. Network analysis based on correlations provides valuable insights into the microbial interactomes and generates hypotheses for understanding oral homeostasis even though correlations are by no means the empirically validated microbial interactions[59].

We further explore the specific enrichment of the function among these distinct microbial communities inhabiting niches and find that the indicative microbial community adapts to specific body sites[9]. Remarkably, we find that there are enriched OTUs associated with activated cell activities signal pathways like cell wall/membrane/envelope biogenesis, cytoskeleton, intracellular trafficking, secretion, and vesicular transport, cell motility et al. in the gingival niche. The marginal gingiva is a $1.5 \mathrm{~mm}$ strip of gingival tissue which surrounds the neck of the tooth. The inner wall forms the gingival wall of the sulcus. This unique microenvironment is full of abundant nutrition, proper temperature, and humidity otc., which is a best natural habitat for colonization and proliferation of a great deal of oral microorganisms. Palate as a masticatory oral mucosa, increases the relative abundance of several metabolic and biosynthetic pathway-related OTUs, which could be argued for the resistance to mechanical damage. Buccal mucosa, activated with replication, recombination, repair, cell cycle control, cell division, chromosome partitioning pathway-related OTUs, is due to the function of lining. And specialized (tongue) oral mucosa raises the relative abundance of amino acid transport and metabolism, nucleotide transport and metabolism, defense mechanisms, translation, ribosomal structure, biogenesis and transcription pathway-related OTUs. Namely, it suggests that the most significant factor that determines the niche for a microbe is its local habitat, which includes its immediate neighbors. It implies that the co-evolution of microbes within the mouth has led to highly specific taxon-taxon interactions that result in most microbes 
being restricted to the habitat type in the mouth that is occupied by those neighbors[8, 14]. Previous study investigating mechanisms among core metabolic pathways of the human microbiome by functionally profiling all HMP1-II samples using the program HUMAnN2[21] also supports our findings[9]. Since habitat refers to externalities -the physical space and chemical environment that allow an organism to exist, including contributions from other members of the microbial community. Niche refers to the activity of an organism and the functional role that each member plays in the community[57]. Indeed, all these results suggest that the niche-specialist hypothesis has important implications for the structure and function of oral microbial communities. Additionally, our evaluation on the oral microbiota of both toothassociated (gingiva), masticatory (palate), lining (buccal), and specialized (tongue) oral mucosa in homeostasis provides a baseline toward assessment to oral mucosal disease associated with these sites, which typically present with unique disease susceptibilities[2].

\section{Evolution of the oral microbiome: the age-related hypothesis}

Time outweighs the effect of host developmental stage on microbial community composition[60]. Our findings reinforce the critical role of ageing in oral microbial composition and functions and suggest that there is a trend for disease-associated taxa to increase and health-associated taxa to decrease with ageing. Notedly, the microbial richness is higher in old mice than that in adult samples, and several opportunistic pathogens such as Stenotrophomonas, Ralstonia, Enterococcus, Bacillaceae otc. are proportionally enriched in old mice, which may be due to the reduction in oral clearance and the local antibodies or antimicrobial peptides. Thinning of the oral mucosa also compromises local mucosal immunity and its antimicrobial capacities $[5,61]$. It also reveals that the distribution in healthy individuals of potentially pathogenic taxa[14]. These examples highlight that with ageing, change in the ecology of the oral cavity is associated with a risk for oral diseases[62]. Preza et al. described the bacterial diversity of different oral niches from older adults without root caries or periodontitis[63]. The most commonly detected species when all sites were evaluated together were Streptococcus oralis, Veillonella atypica, Streptococcus parasanguinis and Fusobacterium nucleatum. The results in their study and this study have emphasized the bacterial profiles of healthy elders might be more diverse than those of young and middle-aged adults.

Each microbe in the mouth is specialized for one life stage or another, so that the microbiota at one period is different from the microbiota at other periods not only in overall composition and proportions of common taxa but also in specific membership. Therefore, network analysis is performed to reveal the modularity of the microbial communities. Individual modules may comprise predominantly health- or disease-associated taxa and may be found in both adult and old samples[14]. All results resolve potential pathogens from nonpathogens that were previously lumped together, and they provide a baseline estimate for the carriage rate and abundance of potential pathogens in oral homeostasis. Inspiringly, the findings that old individuals nevertheless harbor ecological niches that support disease-associated bacteria also support that the shift of health-compatible to disease-inducing microbiome was due to the proportional increases of pathogenic bacteria with ageing, and not due to de novo colonization of disease-associated bacteria in previously healthy individuals[59]. Also, the results suggest that 
antagonism between oral bacteria is a major driving force in the formation of the old-mice oral microbial community.

Intriguingly, increase in metabolic and biogenesis pathway-related OTUs are documented in adult mice, while cell period pathways like cell cycle control, cell division, chromosome partitioning, replication, recombination, and repair pathway-related OTUs, are increased in old mice. During adult life, the composition and proportions of the resident microbes are considered to be reasonably stable, and they coexist in harmony with the host[64]. Physiological changes of ageing include different tissues and functions of the oral cavity[5,61]. As such, the bone density of the jaws is reduced due to loss of calcified tissue and modifications in the collagen structure. It is comprehensible that with ageing, oral mucosa becomes lack of metabolism activities and increasing of tissue repairment, which also reflects in the distinguishable enrichment of oral microorganisms. These works indicate that the host environment drives changes in the oral microbiome over time[65]. The context of the individual's environmental exposure offers an opportunity to predict risk for early disease markers, providing insights into the development of next generation diagnostics. It is not yet known whether any of these ecotypes correlate with increased predisposition to oral disease, which need further study.

\section{The microbiota-immune cell axis in oral homeostasis}

Anther strength of our study is the exploration of immune cell recruitment in different oral niches and ages. Recently, a new concept that the crosstalk between the oral microbiota and mucosal immunity may regulate oral mucosal diseases has been suggested[2]. However, before disease can be fully understood, it is necessary to have a clear understanding of oral homeostasis. Previous studies have shed light on the immunological role of the adult oral epithelium[1]; nevertheless, the spatial and life-long characteristics of immune cells in oral homeostasis remains ill defined. To our knowledge, this is the first study to date to characterize oral recruited mucosal immune cells at four habitats (gingiva, palate, buccal and tongue) as well as during two life stages (adult and old) in oral homeostasis.

In the current study, we elucidate an increase in Th1 cell populations in the setting of adult's tongue and buccal mucosa as well as an increased populations of Th2, Th17 and Treg in the setting of old mice mucosa. Our work here details an atlas of Th cell populations, especially later in life, providing insights into cell functionality in homeostasis and disease susceptibility, opening avenues for further mechanistic exploration of these subsets. Th cell populations are characterized by the production of different effector cytokines and the expression of distinct transcription factors to defense pathogens and maintain balance[2]. Th2 cell differentiation is initiated by T cell receptor (TCR) signal together with IL-4 and subsequently by STAT6 signal transduction, leading to the expression of the transcription factor GATA$3[66]$. Th17 cells are an important subset of effector $T$ cells that are protective during extracellular bacterial and fungal invasion[67-71]. Tregs are essential for maintaining self-tolerance and immunosuppression[72]. As discussed above, due to the reduction in oral clearance and the local antibodies or antimicrobial peptides, several opportunistic pathogens are proportionally enriched in old mice. The increased populations of Th2, Th17 and Treg in the setting of old mice mucosa could be 
suggested to maintain host immune homeostasis and minimize tissue damage. $\gamma \delta T$ cells are also critical mediators of oral mucosal homeostasis. In gingiva, we further document significant recruitment of $ү \delta T$ cells by flow cytometry, as previously reported[39, 73, 74]. $ү \delta T$ cells reside in the gingiva, an oral tissue covered with specialized epithelium that continuously monitors the challenging dental biofilm. Whereas most research on intraepithelial $\gamma \delta T$ cells focuses on the skin, intestine epithelia and gingiva[39, 73-76], our knowledge on these cells in other oral habitats is still incomplete. Importantly, an unreported aspect of the high proportion of $y \delta T$ cells in buccal tissue is the specification of some unique microorganisms toward the recruitment of $\gamma \delta T$ cells. Further study will be carried out for the subsets and functions of these $\gamma \delta T$ cells in buccal tissue.

Finally, we utilize our atlas to explore the correlation between the abundance of the oral microbiomes and the recruited immune cells, providing a further understanding of microbiota-immune axis underlying oral mucosal homeostasis and ageing. The first encounter of mucosal barriers with the microbiota initiates host-microbiota feedback loops instructing the tailored development of both the immune system and microbiota at each mucosal site. Once established, balanced immunological interactions enable symbiotic relationships with the microbiota in adult life[1]. We therefore create a correlation analysis and revealed that Th1 cells perform a negative correlation with strictly anaerobic or facultative anaerobic bacterial, and opportunity infectious bacterial. On the contrary, Treg cells revealed a positive correlation with genus Sporosarcina, Atopostipes and Bradyrhizobium. Furthermore, the abundance of old miceenriched bacterial positively correlated with the proportion of Th2, Th17, and Treg and negatively correlated with the proportion of Th1. These findings suggest a previously unacknowledged role for the age-related microbiota in immune responsiveness in oral homeostasis, similar to recent findings that identify a microbiota-immune axis in ageing of other tissue compartments[4, 77-81]. For example, mucosal colonization of Porphyromonas gingivalis, an important component of the dysbiotic microbiome, can regulate the plasticity of interstitial Th17 and Treg cells leading to an unfavorable balance that promotes disease[82]. Oral mucosal homeostasis is established and shaped by hostmicrobiota interactions in early life[1]. Th cells residing in the mucosa play important roles in mucosal immunity and tolerance[38]. T cell deficiency or defects in T cell function are associated with several oral mucosal diseases[2,72]. Indeed, our results expand the original finding of the phenotype and function of T cell subsets interplaying with the oral microbiota that reside in the oral mucosal homeostasis in old life. Further studies in targeting the specific oral microbiome-immune response and causal mechanisms can conclusively define the role of microbiota in the psychology of oral homeostasis and its contribution to microbial dysbiosis.

Despite our study has several strengths, we also have some shortcomings. Limit to study design, we identify the association between the oral microbiota and several T cell subsets such as Th cells and $y \delta T$ cells. Nevertheless, there are still other immune cells locating in the oral mucosa, and responding to oral commensals and pathogens, such as a unique population of $\mathrm{CD}^{+} \mathrm{CD} 4 / \mathrm{CD} 8$ double-negative $\mathrm{T}$ cells termed mucosa-associated invariant T (MAIT) cells, neutrophils, innate lymphoid cells (ILCs), oct.[1], which are also deserved fully studied in the future. To identify the correlation between the oral inherent 
microbiota and the resided immune cells in different niches and ages in the context of oral mucosal homeostasis, we performed our studies on animal experiments at present. However, animal models will never fully replicate all factors of human biology. There is still a need to conduct clinical studies on humans to gain a holistic understanding of the key features of the oral ecosystem that contribute to oral health. Further research should be carried out to confirm the hypothesis on human samples and reinforce the mechanistic studies on causal relationship as well as cell-specific functionality in disease susceptibility and pathogenesis.

\section{Conclusions}

To our knowledge, this is the first study to date to characterize oral bacterial microbiome communities at four oral niches (tongue, buccal, gingival, and palate) as well as in two major life stages (adult and old) in the context of oral homeostasis and analyze their correlations with immune subsets in oral mucosa. Our findings provide critical insights on microbial communities and species that are niche-specialist and agerelated in the oral cavity, and their potential association with Th cells and $\gamma \delta T$ cells. A clear understanding of microbiota structure at different sites and ages in the mouth enables mechanistic studies, informs the generation of hypotheses, and strengthens the position of oral mucosal microbiology as a model system for microbial ecology in general. Future studies should aim to uncover the co-abundance of specific microbial communities to explore their potential roles in the etiology of microbiota-driven pathogenesis in prospective and longitudinal studies.

\section{Declarations}

\section{Ethics approval and consent to participate}

All experiments were carried out in accordance with institutional guidelines.

\section{Consent for publication}

Not applicable.

\section{Competing interests}

The authors declare that they have no conflict of interest.

\section{Funding}

The research was supported by grants from the National Natural Science Foundations of China (No. 81772896 and 81972532). The authors declare no potential conflicts of interest with respect to the authorship and/or publication of this review.

\section{Authors' contributions}


Dongjia Lin: Formal analysis, Writing-original draft, Supervision, Methodology, Software, Data curation. Lisa Yang, Qiannan Hu: Software, Formal analysis, Visualization, Data curation. Dikan Wang, Wenxiao Dai: Formal analysis, Data curation. Yiwei Xiao, Fang Juan, Hanzi Lu: Writing-review \& editing. Zhi Wang: Conceptualization, Writing-original draft, Writing - review \& editing, Supervision.

\section{Acknowledgements}

The research was supported by grants from the National Natural Science Foundations of China (No. 81772896 and 81972532 ). The authors declare no potential conflicts of interest with respect to the authorship and/or publication of this review.

\section{References}

1. Zubeidat K, Hovav AH. Shaped by the epithelium - postnatal immune mechanisms of oral homeostasis. Trends Immunol. 2021;42:622-34.

2. Lin DJ, Yang LS, Wen LL, Lu HZ, Chen QM, Wang Z. (2021), 'Crosstalk between the oral microbiota, mucosal immunity, and the epithelial barrier regulates oral mucosal disease pathogenesis', Mucosal Immunol.

3. Bergmeier LA. Oral Mucosa in Health and Disease. Cham: Springer, Cham; 2018.

4. Koren N, Zubeidat K, Saba Y, Horev Y, Barel O, Wilharm A, et al. Maturation of the neonatal oral mucosa involves unique epithelium-microbiota interactions. Cell Host Microbe 2021;29:197-209 e5.

5. Belibasakis GN. Microbiological changes of the ageing oral cavity. Arch Oral Biol. 2018;96:230-2.

6. Socransky SS, Manganiello SD. The oral microbiota of man from birth to senility. J Periodontol. 1971;42:485-96.

7. Mark Welch JL, Dewhirst FE, Borisy GG. Biogeography of the oral microbiome: the site-specialist hypothesis. Annu Rev Microbiol. 2019;73:335-58.

8. Mark Welch JL, Ramirez-Puebla ST, Borisy GG. Oral microbiome geography: micron-scale habitat and niche. Cell Host Microbe. 2020;28:160-8.

9. Lloyd-Price J, Mahurkar A, Rahnavard G, Crabtree J, Orvis J, Hall AB, et al. Strains, functions and dynamics in the expanded human microbiome project. Nature. 2017;550:61-6.

10. Libby P. The changing landscape of atherosclerosis. Nature. 2021;592:524-33.

11. Janney A, Powrie F, Mann EH. Host-microbiota maladaptation in colorectal cancer. Nature. 2020;585:509-17.

12. Chen YE, Fischbach MA, Belkaid Y. Skin microbiota-host interactions. Nature. 2018;553:427-36.

13. Simon-Soro A, Tomas I, Cabrera-Rubio R, Catalan MD, Nyvad B, Mira A. Microbial geography of the oral cavity. J Dent Res. 2013;92:616-21.

14. Eren AM, Borisy GG, Huse SM, Mark Welch JL. Oligotyping analysis of the human oral microbiome. Proc Natl Acad Sci U S A. 2014;111:E2875-E84. 
15. Ding R, Liu Y, Yang S, Liu Y, Shi H, Yue X, et al. High-throughput sequencing provides new insights into the roles and implications of core microbiota present in pasteurized milk. Food Res Int. 2020;137:109586.

16. Tropini C, Earle KA, Huang KC, Sonnenburg JL. The gut microbiome: connecting spatial organization to function. Cell Host Microbe. 2017;21:433-42.

17. Proctor DM, Relman DA. The landscape ecology and microbiota of the human nose, mouth, and throat. Cell Host Microbe. 2017;21:421-32.

18. Diaz PI, Valm AM. Microbial interactions in oral communities mediate emergent biofilm properties. J Dent Res. 2020;99:18-25.

19. Valm AM. The structure of dental plaque microbial communities in the transition from health to dental caries and periodontal disease. J Mol Biol. 2019;431:2957-69.

20. Proctor DM, Shelef KM, Gonzalez A, Davis CL, Dethlefsen L, Burns AR, et al. Microbial biogeography and ecology of the mouth and implications for periodontal diseases. Periodontol 2000. 2020;82:2641.

21. Abubucker S, Segata N, Goll J, Schubert AM, Izard J, Cantarel BL, et al. Metabolic reconstruction for metagenomic data and its application to the human microbiome. PLoS Comput Biol. 2012;8:e1002358.

22. Yang F, Zeng X, Ning K, Liu KL, Lo CC, Wang W, et al. Saliva microbiomes distinguish caries-active from healthy human populations. ISME J. 2012;6:1-10.

23. Huang S, Li R, Zeng XW, He T, Zhao HL, Chang A, et al. Predictive modeling of gingivitis severity and susceptibility via oral microbiota. ISME J. 2014;8:1768-80.

24. Partridge L, Deelen J, Slagboom PE. Facing up to the global challenges of ageing. Nature. 2018;561:45-56.

25. Campisi J, Kapahi P, Lithgow GJ, Melov S, Newman JC, Verdin E. From discoveries in ageing research to therapeutics for healthy ageing. Nature. 2019;571:183-92.

26. Cavalli G, Heard E. Advances in epigenetics link genetics to the environment and disease. Nature. 2019;571:489-99.

27. Schumacher B, Pothof J, Vijg J, Hoeijmakers JHJ. The central role of DNA damage in the ageing process. Nature. 2021;592:695-703.

28. Ezzati M, Pearson-Stuttard J, Bennett JE, Mathers CD. Acting on non-communicable diseases in lowand middle-income tropical countries. Nature. 2018;559:507-16.

29. Pega F, Nafradi B, Momen NC, Ujita Y, Streicher KN, Pruss-Ustun AM, et al. Global, regional, and national burdens of ischemic heart disease and stroke attributable to exposure to long working hours for 194 countries, 2000-2016: a systematic analysis from the WHO/ILO joint estimates of the workrelated burden of disease and injury. Environ Int. 2021;154:106595.

30. Yatsunenko T, Rey FE, Manary MJ, Trehan I, Dominguez-Bello MG, Contreras M, et al. Human gut microbiome viewed across age and geography. Nature. 2012;486:222-+. 
31. Feres $M$, Teles F, Teles R, Figueiredo LC, Faveri M. The subgingival periodontal microbiota of the aging mouth. Periodontol 2000. 2016;72:30-53.

32. Ebersole JL, Dawson DA 3rd, Emecen Huja P, Pandruvada S, Basu A, Nguyen L, et al. Age and periodontal health - immunological view. Curr Oral Health Rep. 2018;5:229-41.

33. Zemouri C, Ofiteru ID, Jakubovics NS. Future directions for studying resilience of the oral ecosystem. Br Dent J. 2020;229:769-73.

34. Dutzan N, Konkel JE, Greenwell-Wild T, Moutsopoulos NM. Characterization of the human immune cell network at the gingival barrier. Mucosal Immunol. 2016;9:1163-72.

35. Sharawi H, Heyman O, Mizraji G, Horev Y, Laviv A, Shapira L, et al. The prevalence of gingival dendritic cell subsets in periodontal patients. J Dent Res. 2021;26:220345211004864.

36. Gaffen SL, Moutsopoulos NM. Regulation of host-microbe interactions at oral mucosal barriers by type 17 immunity. Science Immunology. 2020;5:eaau4594.

37. Hovav AH, Wilharm A, Barel O, Prinz I. Development and function of gammadeltaT cells in the oral mucosa. J Dent Res. 2020;99:498-505.

38. Wu RQ, Zhang DF, Tu E, Chen QM, Chen W. The mucosal immune system in the oral cavity-an orchestra of T cell diversity. Int J Oral Sci. 2014;6:125-32.

39. Wilharm A, Tabib Y, Nassar M, Reinhardt A, Mizraji G, Sandrock I, et al. Mutual interplay between IL17-producing gamma delta $T$ cells and microbiota orchestrates oral mucosal homeostasis. Proceedings of the national academy of sciences of the United States of America 2019;116:2652-61.

40. Seo GY, Giles DA, Kronenberg M. The role of innate lymphoid cells in response to microbes at mucosal surfaces. Mucosal Immunol. 2020;13:399-412.

41. Lin QC, Kuypers M, Philpott DJ, Mallevaey T. The dialogue between unconventional T cells and the microbiota. Mucosal Immunol. 2020;13:867-76.

42. Kayama H, Okumura R, Takeda K. Interaction between the microbiota, epithelia, and immune cells in the intestine. Annu Rev Immunol. 2020;38:23-48.

43. Domingue JC, Drewes JL, Merlo CA, Housseau F, Sears CL. Host responses to mucosal biofilms in the lung and gut. Mucosal Immunol. 2020;13:413-22.

44. Van Dyke TE, vWAJ. Infection and infammatory mechanisms. J Periodontol. 2013;84:1-7.

45. Sundberg JP, Berndt A, Sundberg BA, Silva KA, Kennedy V, Smith RS, et al. Approaches to investigating complex genetic traits in a large-scale inbred mouse aging study. Vet Pathol. 2016;53:456-67.

46. Snyder JM, Ward JM, Treuting PM. Cause-of-death analysis in rodent aging studies. Vet Pathol. 2016;53:233-43.

47. Ward JM, Youssef SA, Treuting PM. Why animals die: an introduction to the pathology of aging. Vet Pathol. 2016;53:229-32.

48. Ackert-Bicknell CL, Anderson LC, Sheehan S, Hill WG, Chang B, Churchill GA, Chesler EJ, Korstanje R. and L. L. Peters. Aging research using mouse models. Curr Protoc Mouse Biol. 2015;5:95-133. 
49. Treuting PM, Suzanne M, Dintzis CW, Frevert H, Denny Liggitt, Kathleen S. Montine. Comparative anatomy and Histology: A Mouse and Human Atlas. Elsevier/Academic Press: Amsterdam ; Boston; 2012.

50. Grubb SC, Bult CJ, Bogue MA. Mouse phenome database. Nucleic Acids Res. 2014;42:D825-0834.

51. Flurkey K, Currer JM. and D. E. Harrison.. The Mouse in Biomedical Research in Fox JG, editor. Amsterdam ; Boston: American College of Laboratory Animal Medicine series 2007.

52. Clausen BE, Laman JD, Inflammation. Mainz, Germany: Humana Press, 2017.

53. Rautava J, Pinnell LJ, Vong L, Akseer N, Assa A, Sherman PM. Oral microbiome composition changes in mouse models of colitis. J Gastroenterol Hepatol. 2015;30:521-7.

54. Caporaso JG, Kuczynski J, Stombaugh J, Bittinger K, Bushman FD, Costello EK, et al. QIIME allows analysis of high-throughput community sequencing data. Nat Methods. 2010;7:335-6.

55. Scott CL, Bain CC, Mowat AM. Isolation and identification of intestinal myeloid cells. Methods Mol Biol. 2017;1559:223-39.

56. Daniel N, Lecuyer E, Chassaing B. (2021), 'Host/microbiota interactions in health and diseases-time for mucosal microbiology!', Mucosal Immunol.

57. J W. Spatial order in microbial ecosystems. Biol Rev 1981;56:295-342.

58. Zhou J, Deng Y, Luo F, He Z, Yang Y. Phylogenetic molecular ecological network of soil microbial communities in response to elevated C02. mBio 2011;2:e00122-11.

59. Chen C, Hemme C, Beleno J, Shi ZJ, Ning DL, Qin YJ, et al. Oral microbiota of periodontal health and disease and their changes after nonsurgical periodontal therapy. ISME J. 2018;12:1210-24.

60. Dibner RR, Weaver AM, Brock MT, Custer GF, Morrison HG, Maignien L, et al. 'Time outweighs the effect of host developmental stage on microbial community composition'. FEMS Microbiol Ecol; 2021.

61. Thompson LA, Chen H. Physiology of aging of older adults: systemic and oral health considerations2021 update. Dent Clin North Am. 2021;65:275-84.

62. Santoro A, Zhao JC, Wu L, Carru C, Biagi E, Franceschi C. Microbiomes other than the gut: inflammaging and age-related diseases. Seminars in Immunopathology. 2020;42:589-605.

63. Preza D, Olsen I, Willumsen T, Boches SK, Cotton SL, Grinde B, et al. Microarray analysis of the microflora of root caries in elderly. European Journal of Clinical Microbiology Infectious Diseases. 2009;28:509-17.

64. Mosaddad SA, Tahmasebi E, Yazdanian A, Rezvani MB, Seifalian A, Yazdanian M, et al. Oral microbial biofilms: an update. Eur J Clin Microbiol Infect Dis. 2019;38:2005-19.

65. Freire M, Moustafa A, Harkins DM, Torralba MG, Zhang Y, Leong P, et al. Longitudinal study of oral microbiome variation in twins. Sci Rep. 2020;10:7954.

66. Ouyang W, Ranganath SH, Weindel K, Bhattacharya D, Murphy TL, Sha WC, et al. Inhibition of Th1 development mediated by GATA-3 through an IL-4-independent mechanism. Immunity. 1998;9:74555. 
67. Pandiyan P, Conti HR, Zheng L, Peterson AC, Mathern DR, Hernandez-Santos N, et al. CD4(+)CD25(+)Foxp3(+) regulatory T cells promote Th17 cells in vitro and enhance host resistance in mouse Candida albicans Th17 cell infection model. Immunity. 2011;34:422-34.

68. Conti HR, Shen F, Nayyar N, Stocum E, Sun JN, Lindemann MJ, et al. Th17 cells and IL-17 receptor signaling are essential for mucosal host defense against oral candidiasis. J Exp Med. 2009;206:299-311.

69. Allam JP, Duan Y, Winter J, Stojanovski G, Fronhoffs F, Wenghoefer M, et al. Tolerogenic T cells, Th1/Th17 cytokines and TLR2/TLR4 expressing dendritic cells predominate the microenvironment within distinct oral mucosal sites. Allergy. 2011;66:532-9.

70. Cheng SC, van de Veerdonk FL, Lenardon M, Stoffels M, Plantinga T, Smeekens S, et al. The dectin$1 /$ inflammasome pathway is responsible for the induction of protective T-helper 17 responses that discriminate between yeasts and hyphae of Candida albicans. J Leukoc Biol. 2011;90:357-66.

71. Bhaskaran N, Liu ZH, Saravanamuthu SS, Yan CH, Hu Y, Dong LJ, et al. Identification of Casz1 as a regulatory protein controlling $\mathrm{T}$ helper cell differentiation, inflammation, and immunity. Frontiers in Immunol. 2018;9:184.

72. Zhang YJ, Guo JH, Jia R. Treg: a promising immunotherapeutic target in oral diseases. Frontiers in Immunol. 2021;12:667862.

73. Moutsopoulos NM, Konkel JE. Tissue-specific immunity at the oral mucosal barrier. Trends in Immunol. 2018;39:276-87.

74. Krishnan S, Prise IE, Wemyss K, Schenck LP, Bridgeman HM, McClure FA, et al. Amphiregulinproducing gamma delta $T$ cells are vital for safeguarding oral barrier immune homeostasis. Proc Natl Acad Sci USA. 2018;115:10738-43.

75. Mayassi T, Ladell K, Gudjonson H, McLaren JE, Shaw DG, Tran MT, et al. Chronic inflammation permanently reshapes tissue-resident immunity in celiac disease. Cell 2019;176:967-81 e19.

76. Nielsen MM, Witherden DA, Havran WL. Gammadelta T cells in homeostasis and host defence of epithelial barrier tissues. Nat Rev Immunol. 2017;17:733-45.

77. Baruch KDA, David E, Castellano JM, Miller O, Kertser A, Berkutzki T, Barnett-Itzhaki Z, Bezalel D, Wyss-Coray T, Amit I, Schwartz M. Aging-induced type I interferon response at the choroid plexus negatively affects brain function. Science. 2014;346:89-93.

78. Schwartz M, Peralta Ramos JM, Ben-Yehuda H. A 20-Year Journey from axonal injury to neurodegenerative diseases and the prospect of immunotherapy for combating Alzheimer's disease. J Immunol. 2020;204:243-50.

79. Villeda SA, Luo J, Mosher KI, Zou B, Britschgi M, Bieri G, et al. The ageing systemic milieu negatively regulates neurogenesis and cognitive function. Nature. 2011;477:90-4.

80. Garcia-Pena C, Alvarez-Cisneros T, Quiroz-Baez R, Friedland RP. Microbiota and aging. a review and commentary. Arch Med Res. 2017;48:681-9.

81. Spychala MS, Venna VR, Jandzinski M, Doran SJ, Durgan DJ, Ganesh BP, et al. Age-related changes in the gut microbiota influence systemic inflammation and stroke outcome. Ann Neurol. 2018;84:23- 
36.

82. Curtis MA. The mouth opens wide onto an additional view of immune development at mucosal borders. Cell Host Microbe. 2021;29:148-9.

\section{Figures}
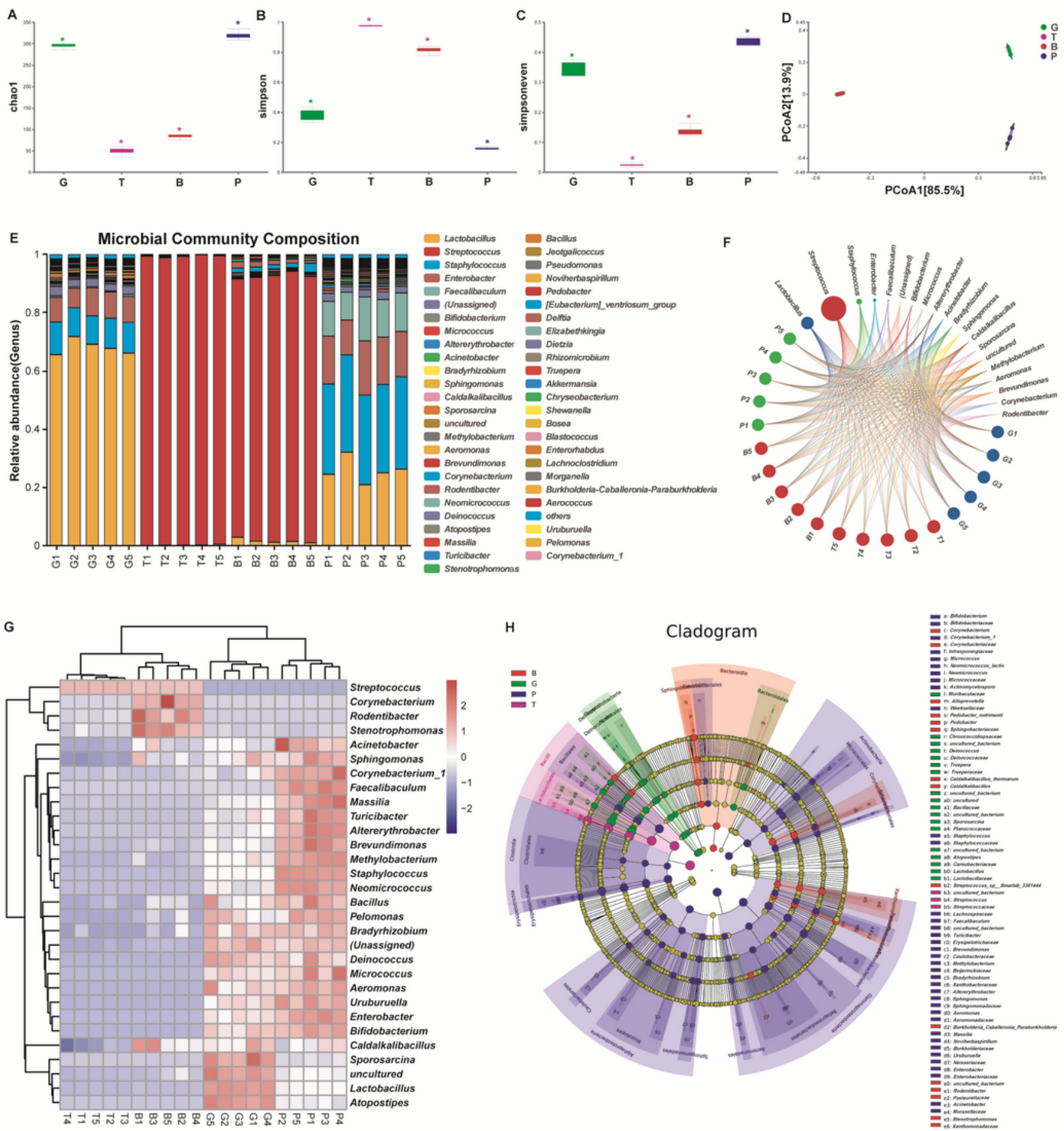


\section{Figure 1}

The oral microbiota community is niche-specialist A-D. Comparative alpha diversity (A-C) and beta diversity (D. Principal coordinate analysis, PCoA) analysis of the oral microbiome in different oral sites. A. Box plot of community richness analysis (chao1 index). B. Box plot of community diversity analysis (simpson index). C. Box plot of community evenness analysis (simpsoneven index). D. Bray-curtis PCoA plot. Each point represents gingiva (green), tongue (pink), buccal (red), and palate (blue) for each saliva sample. E. Bar graph represents the relative community composition at the genus level of the oral microbiota in each oral site. F. Circular layout of the top microbial features at the genus level with each sample. The size of each bacterial point represents the relative abundance of OTUs in all microbial communities. The color of each sample point represents the highest abundance species in it. G. Richness heatmap of the microbiota composition at the genus level within each sample (horizontal axis).

Dendrogram of sample sites based on the community similarity along the left axis. The color of the spots represents the relative abundance of the OTUs. The genus-level taxonomic assignment is shown on the right. Each column represents one subject. $\mathrm{H}$. Taxonomic cladogram obtained from LEfSe analysis of $16 \mathrm{~S}$ sequences, taxonomic representation of statistically significant differences in gingiva (green), tongue (pink), buccal (red) and palate (blue). The diameter of each circle is proportional to taxon abundance. 

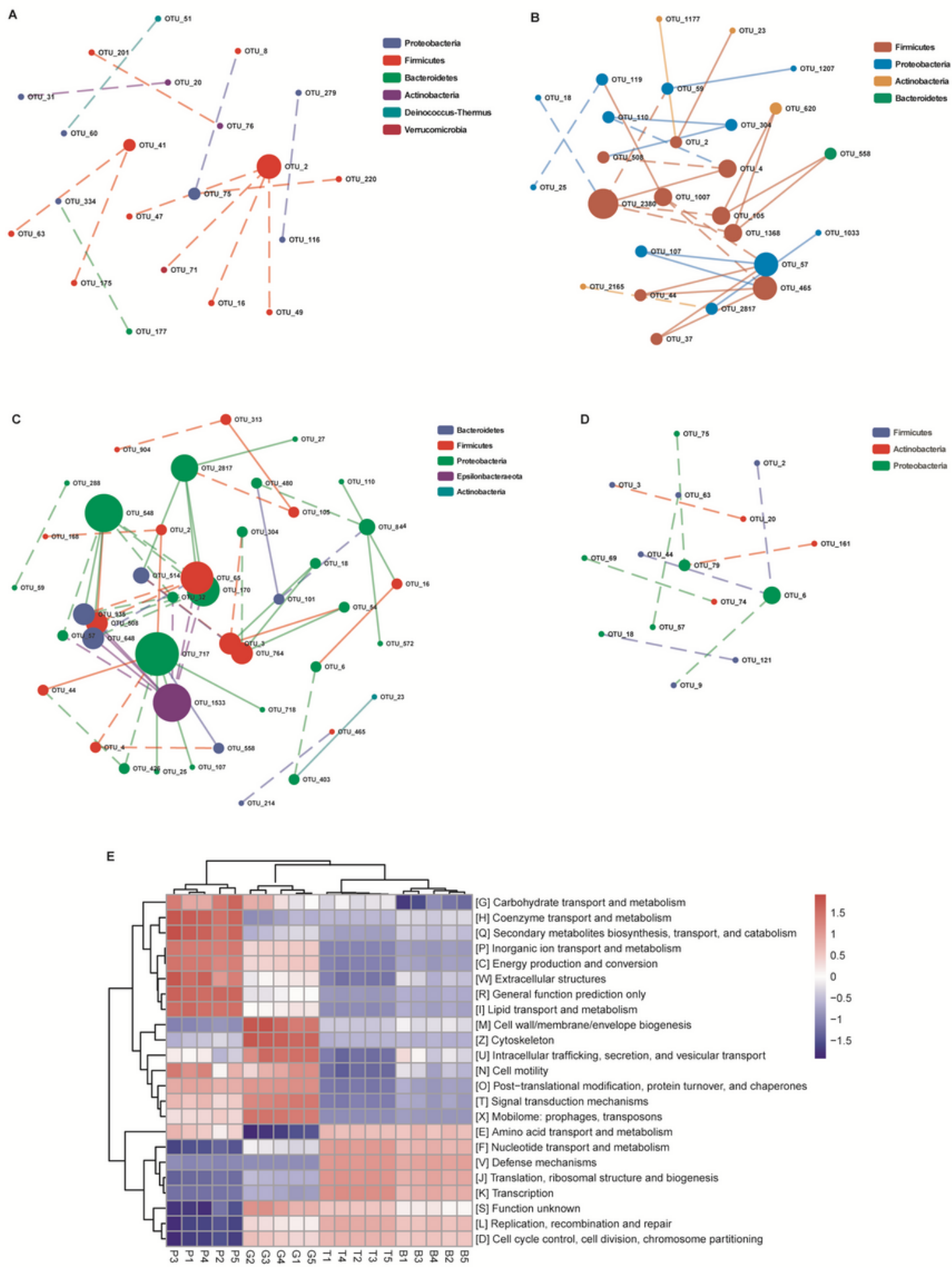

\section{Figure 2}

Taxon-taxon relationship and functional analysis of different communities A. Network analysis for gingival sample. B. Network analysis for tongue sample. C. Network analysis for buccal sample. D. Network analysis for palatal sample. The network analysis was performed at the level of top 50 OTU. The OTU nodes under each genus are merged into the single genus nodes, which are color coded by phyla. The size of the node correlates with the number of links of the node. A solid line indicates a positive 
correlation. The dotted line shows a negative correlation. E. COG functional pathway abundance analysis of the oral microbiota in different habitats. G, G1-5: gingiva, T, T1-5: tongue, B, B1-5: buccal, P, P1-5:

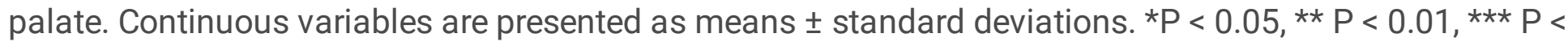
0.001 . Significant differences between groups were analyzed with the Kruskal-Wallis Test.

A
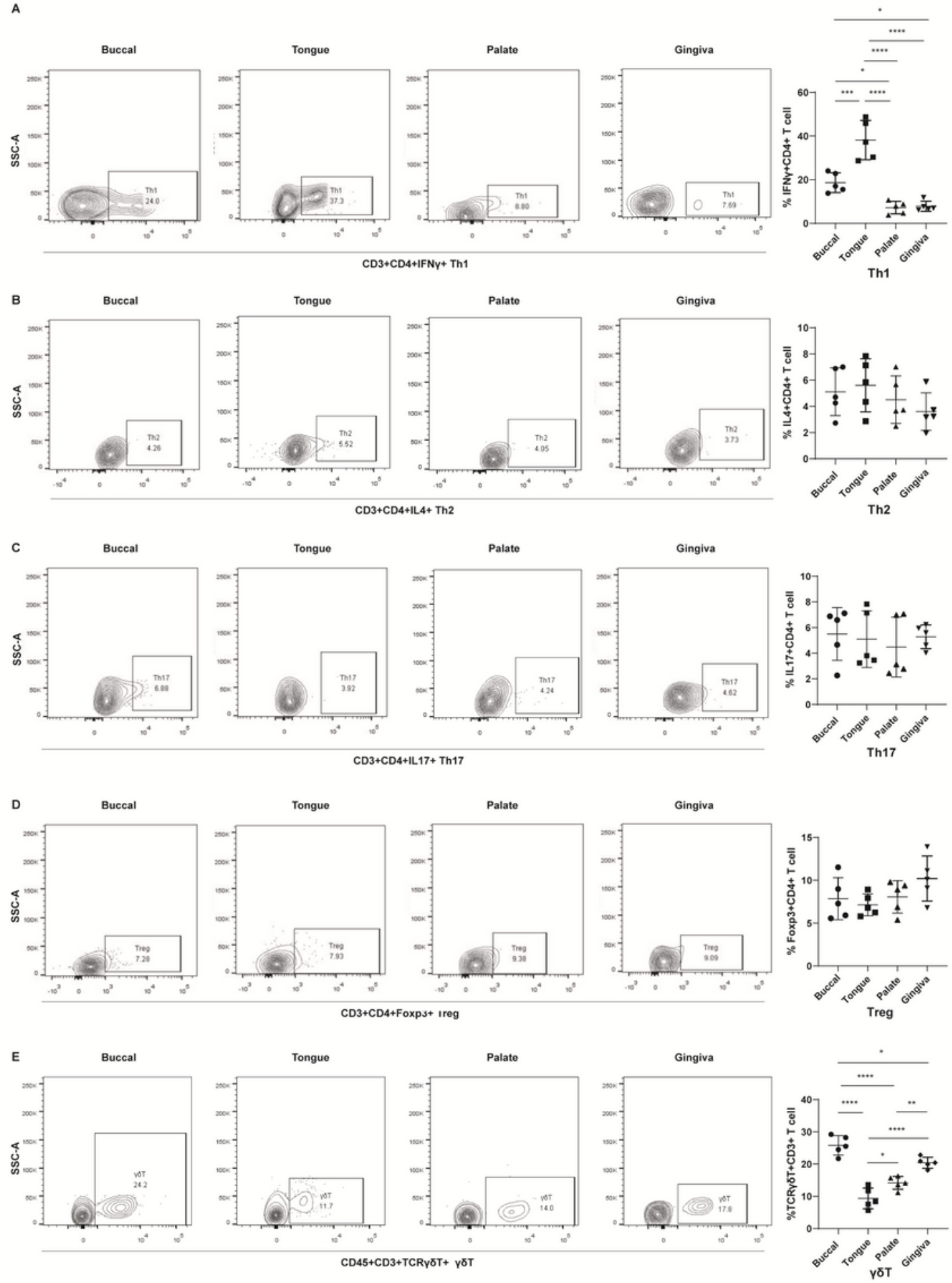

Figure 3 
The abundance of Th1 cells and $\gamma \delta T$ cells distributed distinctive in different oral sites A. Representative flow cytometry data and statistical analysis for IFNy expression in different oral sites. B. Representative flow cytometry data and statistical analysis for IL4 expression in different oral sites. C. Representative flow cytometry data and statistical analysis for IL17 expression in different oral sites. D. Representative flow cytometry data and statistical analysis for Foxp3 expression in different oral sites. E. Representative flow cytometry data and statistical analysis for TCRYठT expression in different oral sites. Continuous variables are presented as means \pm standard deviations. ${ }^{*} P<0.05$, ${ }^{\star *} P<0.01$, ${ }^{\star \star *} P<0.001$. Significant differences between groups were analyzed with the One-way ANOVA.

A
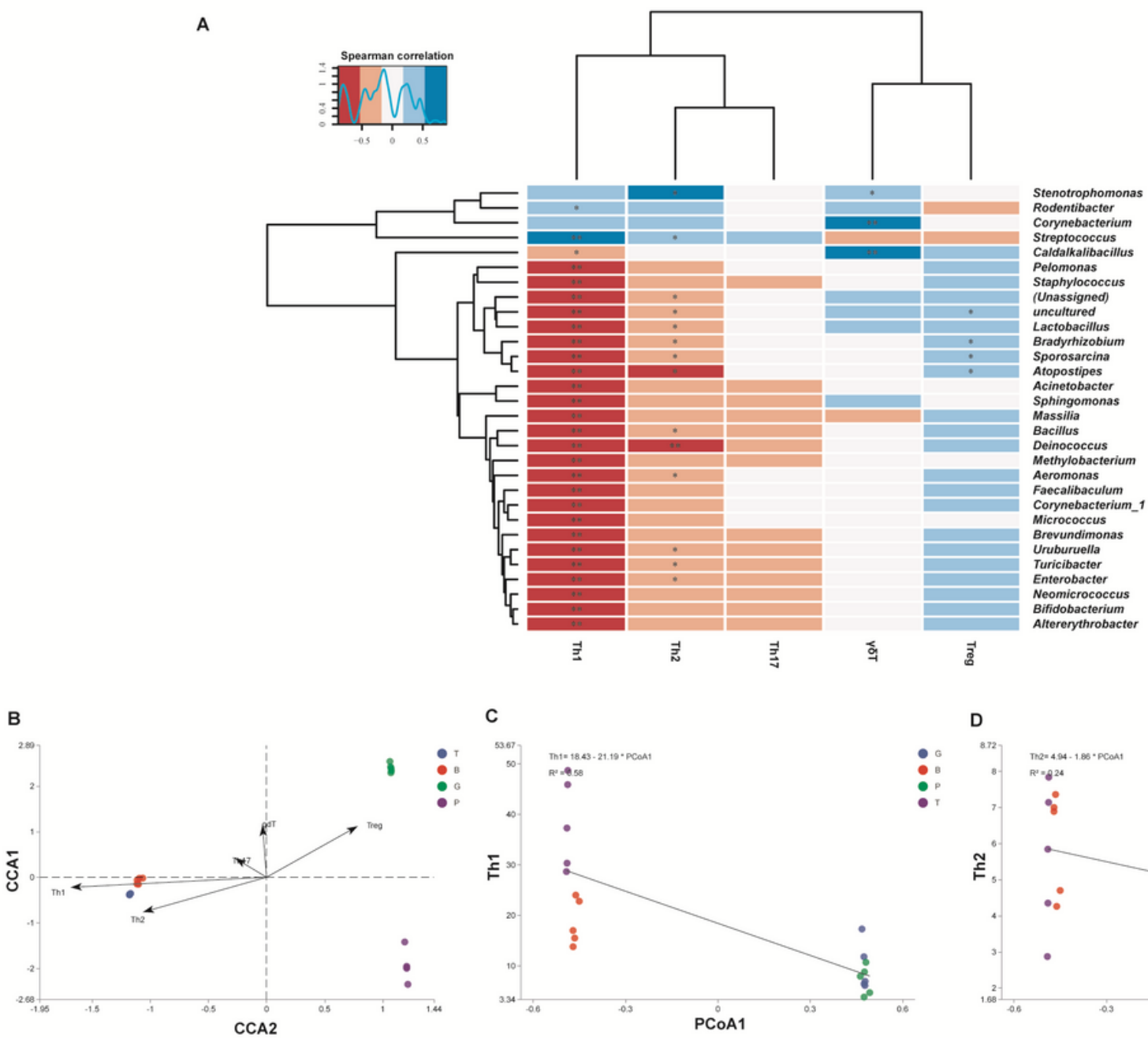

C
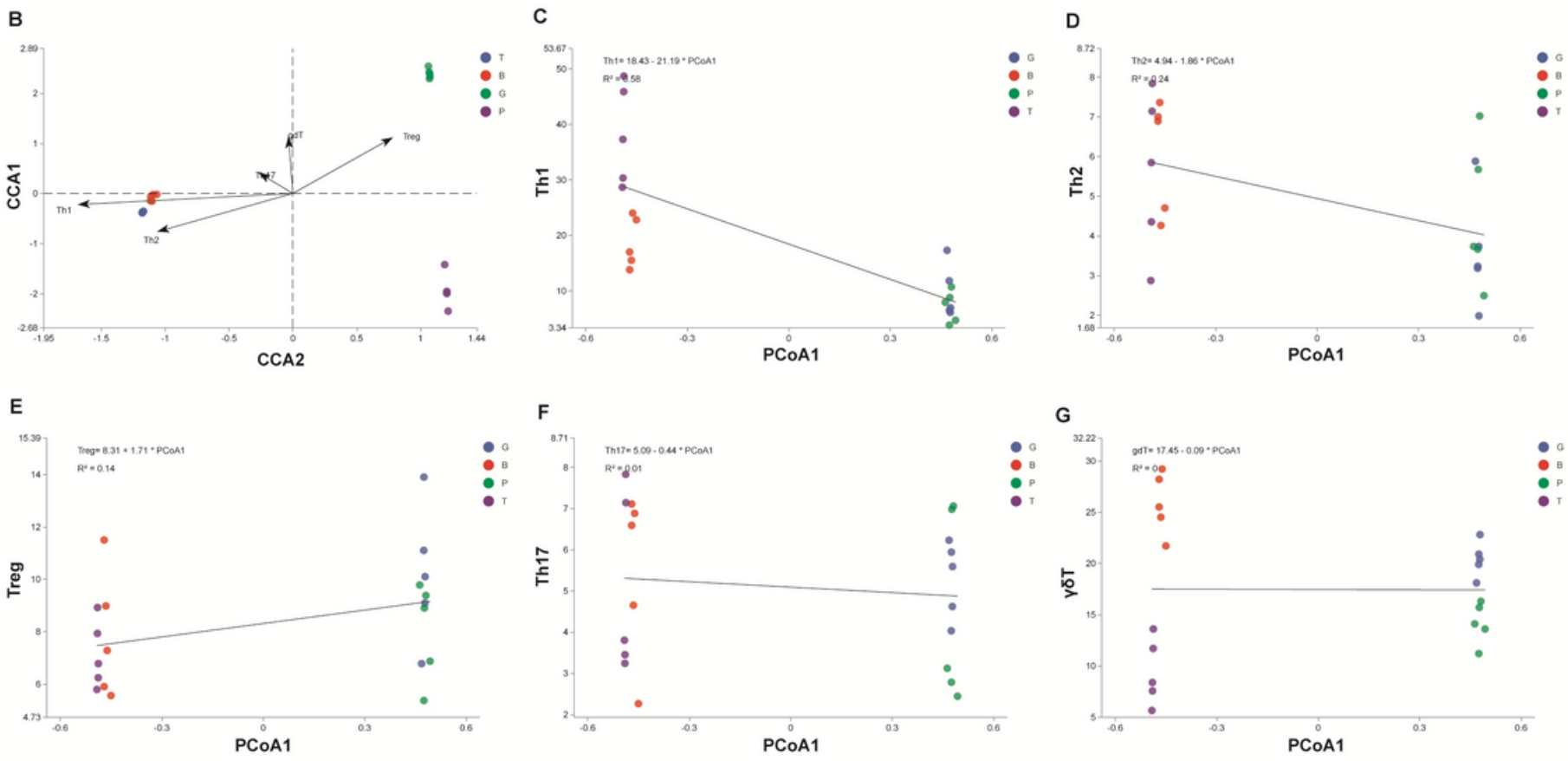

Figure 4 
Correlation analysis between the immune cells and the relative abundance of oral microbiota A. Spearman correlation heatmap. B. CCA analysis. C-G. Linear regression model. Spearman correlation coefficients are represented by color ranging from blue (negative correlation) to red (positive correlation). $* \mathrm{P}<0.05$ and $* * \mathrm{P}<0.01$ based on Mann-Whitney test.
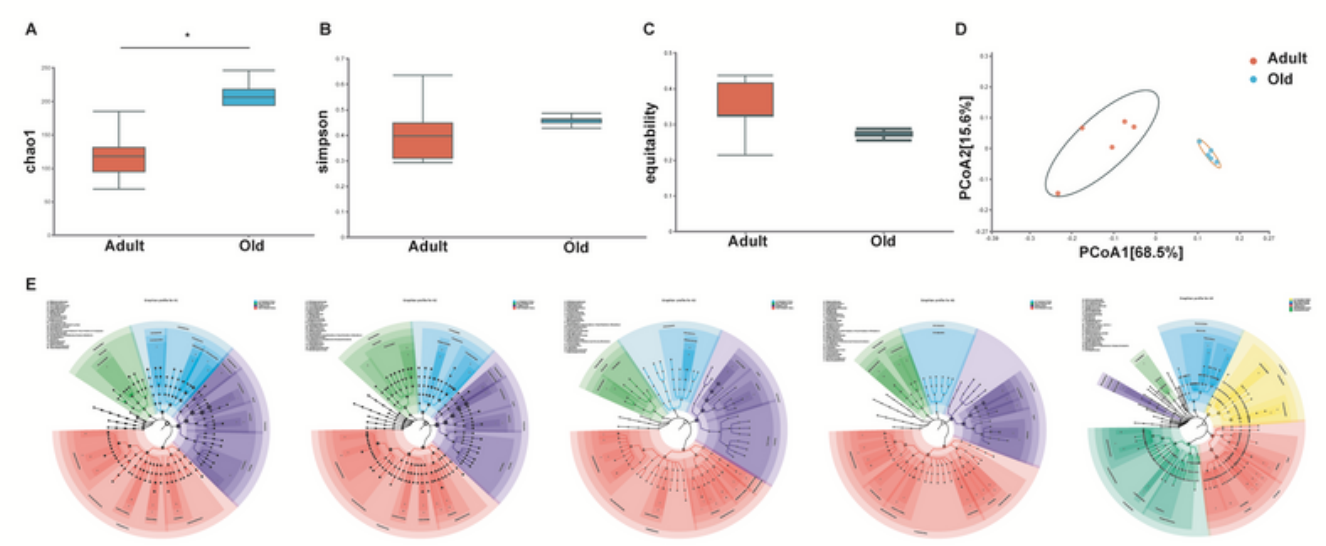

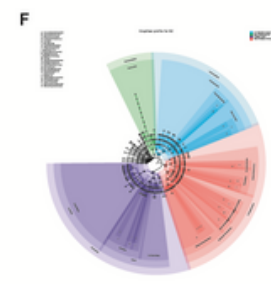

G

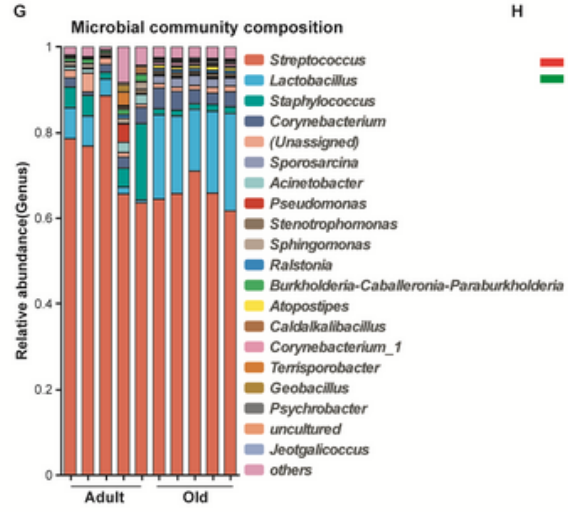

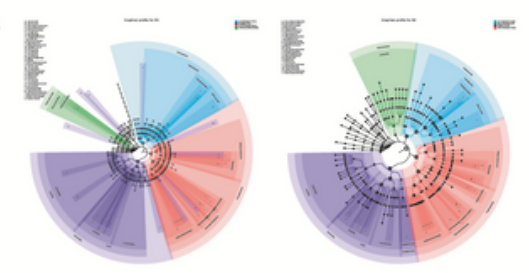

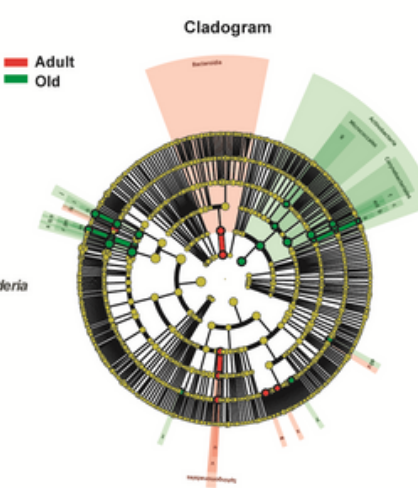

I
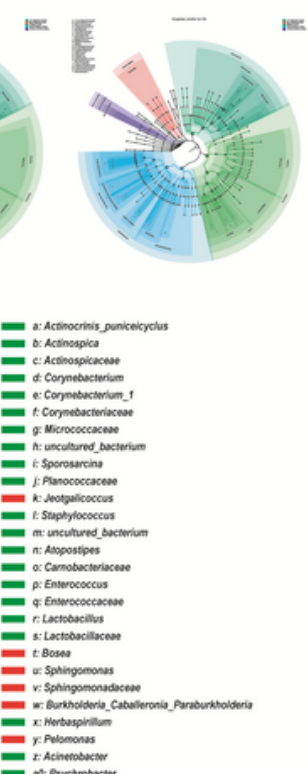

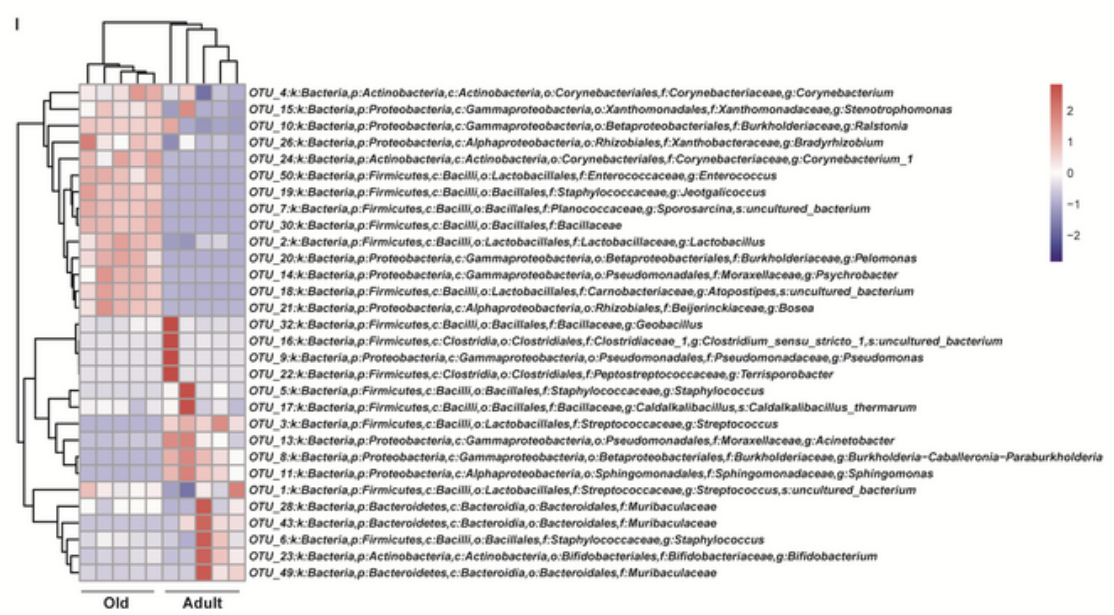

Figure 5 
The oral microbiota community is age-related A-D. Comparative alpha diversity (A-C) and beta diversity (D. Principal coordinate analysis, PCOA) analysis of the saliva microbiome between adult mice and old mice. A. Community richness analysis (chao1 index). B. Community diversity analysis (simpson index). C. Community evenness analysis (simpsoneven index). D. Bray-curtis PCoA plot. Each point represents adult (red) and old (blue) for each saliva sample. E-F. Compact visualizations of microbial metagenomes using Graphical phylogenetic analysis (GraPhIAn) of each sample. E. GraPhIAn profile for adult mice saliva samples. F. GraPhIAn profile for old mice saliva samples. G. Bar graph represents the relative community composition at the genus level in saliva samples of adult mice and old mice. H. Taxonomic cladogram obtained from LEfSe analysis of $16 \mathrm{~S}$ sequences, taxonomic representation of statistically significant differences between adult mice and old mice (red, adult mice; green, old mice). The diameter of each circle is proportional to taxon abundance. I. Richness heatmap of the saliva microbiota composition at the genus level within each sample (horizontal axis) from adult mice and old mice. Dendrogram of sample sites based on the community similarity along the left axis. The color of the spots represents the relative abundance of the OTUs. The genus-level taxonomic assignment is shown on the right. Each column represents one subject. 
A

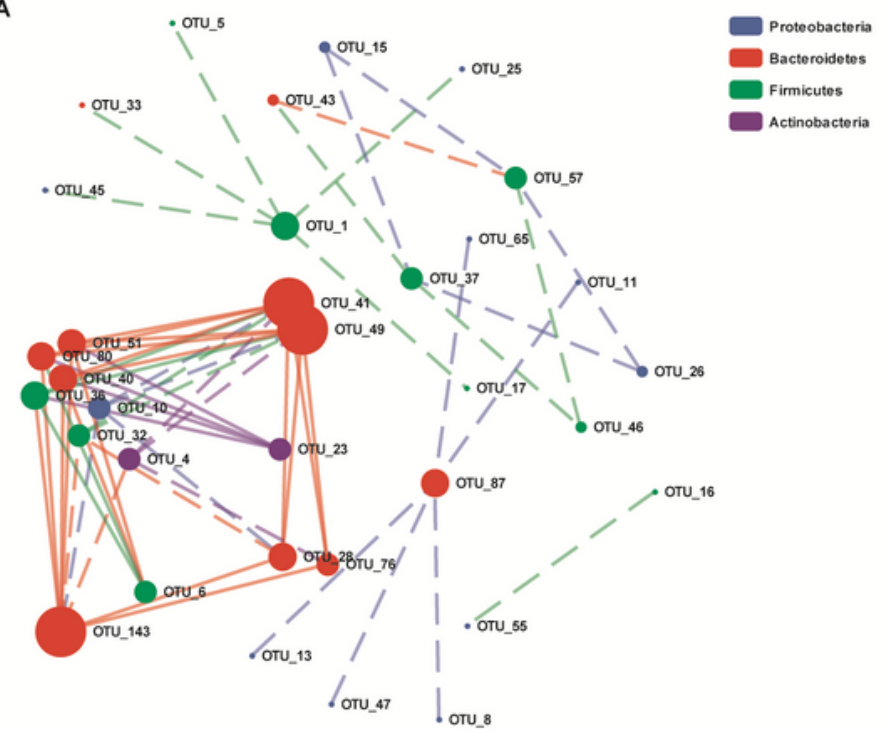

C
B

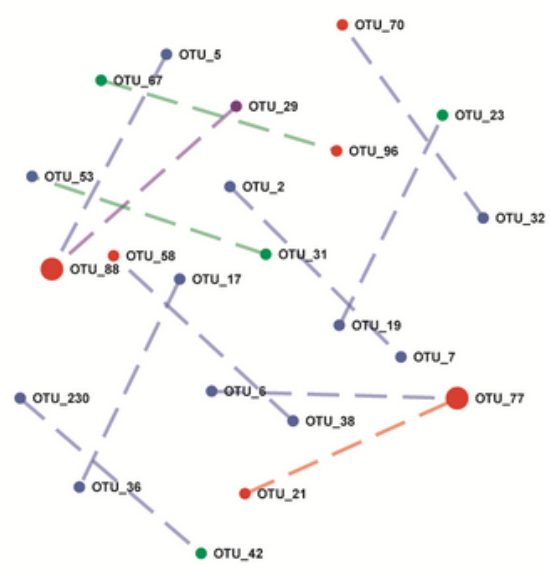

Firmicutes

Protoobacteria

Actinobactoria

Verrucomicrobia

[V] Defense mechanisms $\quad 0.6$

[N] Cell motility

[C] Energy production and conversion $\quad 0.4$

[l] Lipid transport and metabolism $\quad 0.2$

[E] Amino acid transport and metabolism

[P] Inorganic ion transport and metabolism

[Q] Secondary metabolites biosynthesis, transport, and catabolism

[U] Intracellular trafficking, secretion, and vesicular transport

[R] General function prediction only

[H] Coenzyme transport and metabolism

[M] Cell wall/membrane/envelope biogenesis

[O] Post-translational modification, protein turnover, and chaperones

[J] Translation, ribosomal structure and biogenesis

[S] Function unknown

[F] Nucleotide transport and metabolism

[L] Replication, recombination and repair

[X] Mobilome: prophages, transposons

[Z] Cytoskeleton

[D] Cell cycle control, cell division, chromosome partitioning

[T] Signal transduction mechanisms

[G] Carbohydrate transport and metabolism

[K] Transcription

를을

\section{Figure 6}

Taxon-taxon relationship and functional analysis of different stages A. Network analysis for adult sample. B. Network analysis for old sample. The network analysis was performed at the level of top 50 OTU. The OTU nodes under each genus are merged into the single genus nodes, which are color coded by phyla. The size of the node correlates with the number of links of the node. A solid line indicates a positive correlation. The dotted line shows a negative correlation. C. COG functional pathway abundance 
analysis. Continuous variables are presented as means \pm standard deviations. ${ }^{*} P<0.05,{ }^{\star *} P<0.01$, ${ }^{\star \star \star} P$ $<0.001$. Significant differences between groups were analyzed with the Kruskal-Wallis Test.

A
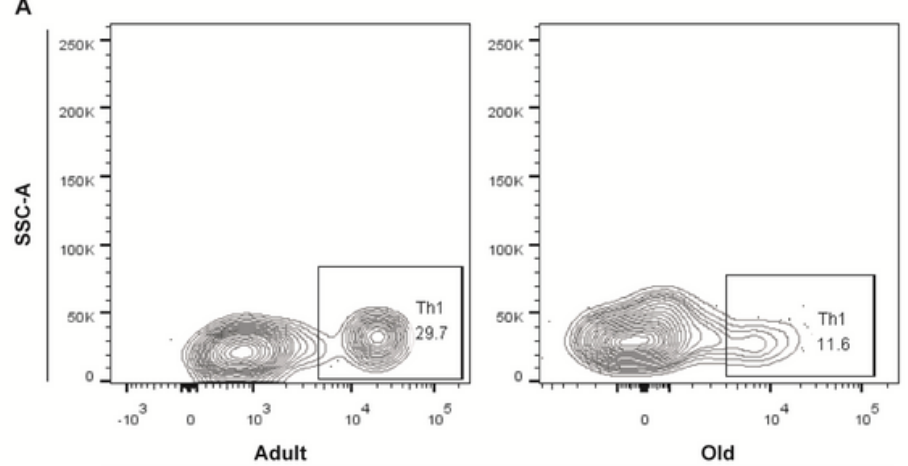

IFNy+CD4+ Th1 cell
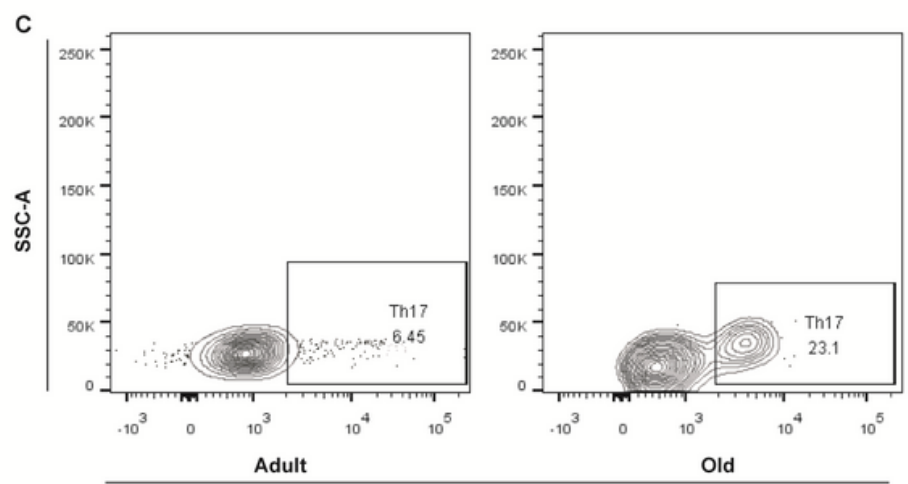

IL17+CD4+ Th17 cell

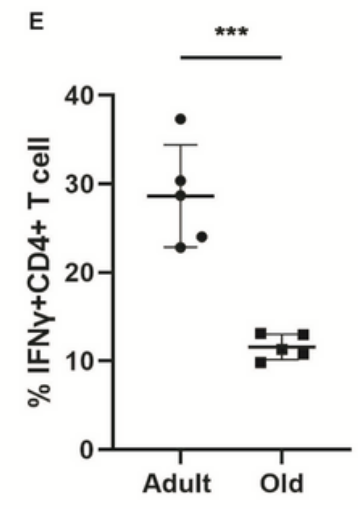

Th1
F

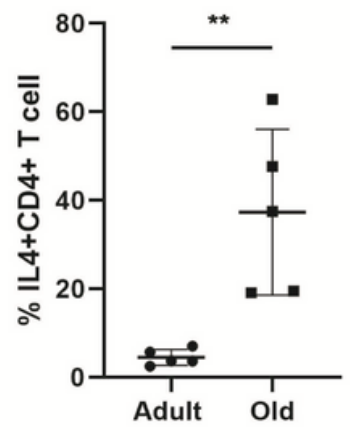

Th2
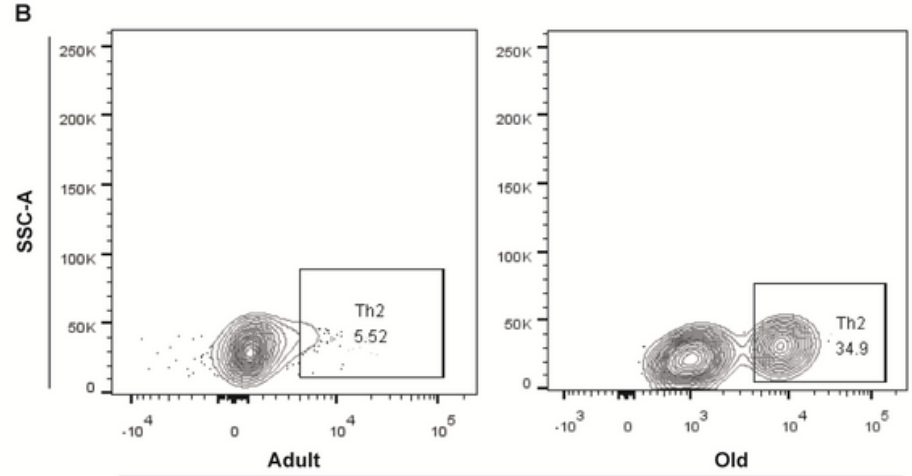

IL4+CD4+ Th2 cell

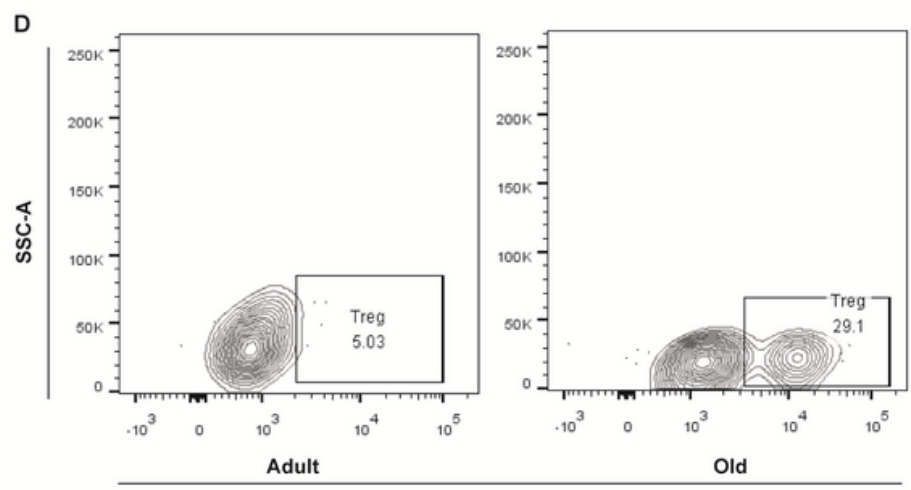

Foxp3+CD4+ Treg cell

G

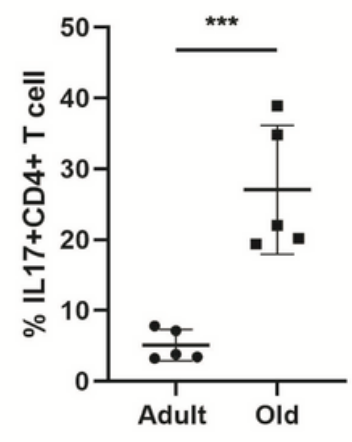

Th17
H

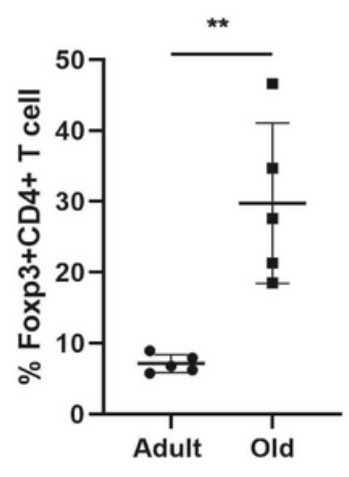

Treg

\section{Figure 7}

The abundance of $\mathrm{CD} 4+\mathrm{T}$ cell subsets distributed distinctive in different life stages A. Representative flow cytometry data for IFNy expression. B. Representative flow cytometry data for IL4 expression. C. Representative flow cytometry data for IL17 expression. D. Representative flow cytometry data for Foxp3 expression. E-H. Statistical analysis. Continuous variables are presented as means \pm standard deviations. $\star P<0.05,{ }^{*} P<0.01$, $* \star * P<0.001$. Significant differences between groups were analyzed with the $T$ test. 
A
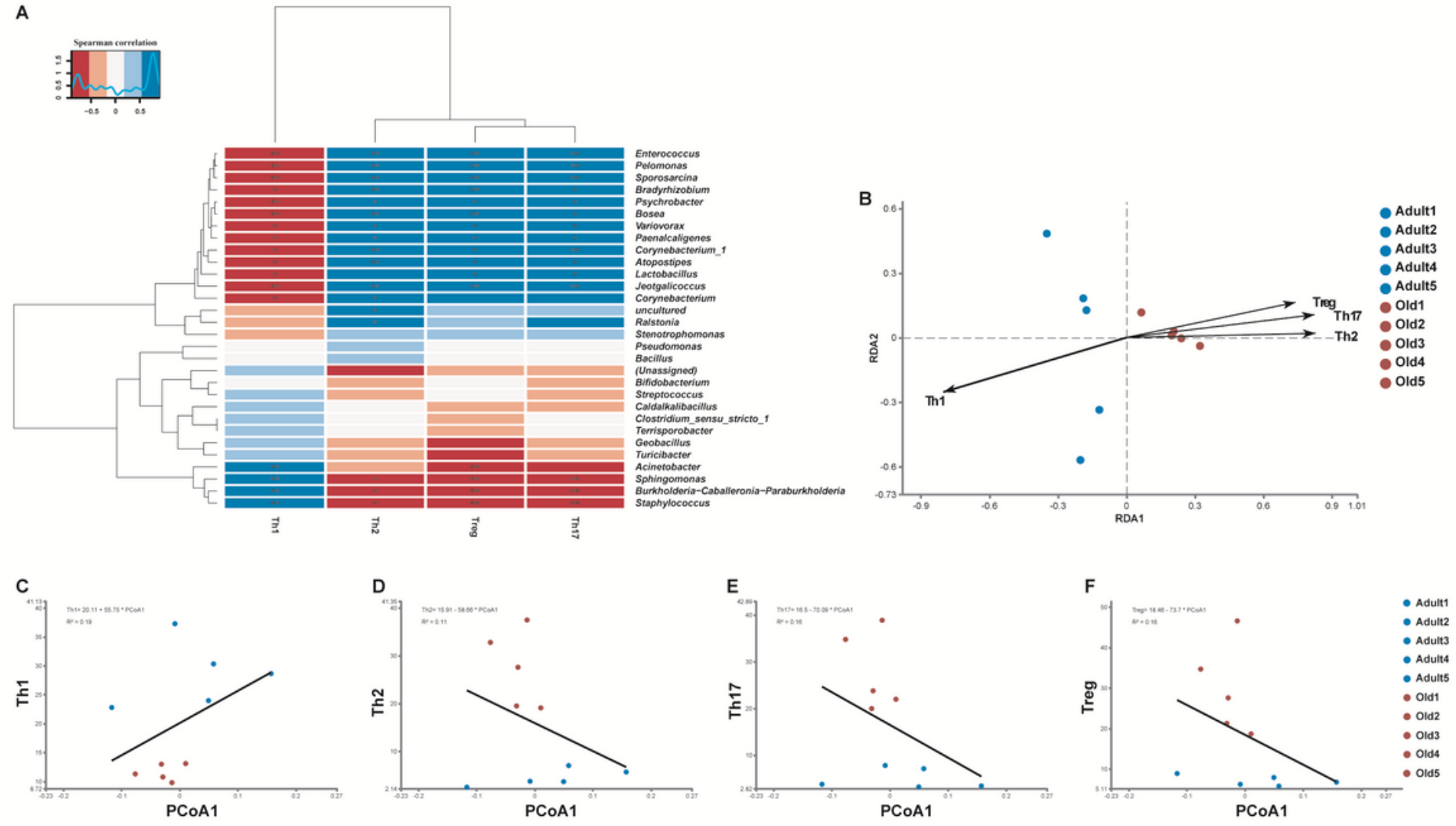

Figure 8

Correlation analysis between the immune cells and the relative abundance of oral microbiota in different life stages A. Spearman correlation heatmap. B. RDA analysis. C-F. Linear regression model. Spearman correlation coefficients are represented by color ranging from blue (negative correlation) to red (positive correlation). ${ }^{*} \mathrm{P}<0.05$ and ${ }^{*} \mathrm{P}<0.01$ based on Mann-Whitney test.

\section{Supplementary Files}

This is a list of supplementary files associated with this preprint. Click to download.

- Fig1sup.tif

- 4.SupplementaryText.doc

- Fig2sup.tif

- Fig3Sup.tif

- Fig4Sup.tif

- Fig5sup.tif 